Accepted by ApJ Letters April 2000

\title{
The Missing Link: Early Methane ("T") Dwarfs in the Sloan Digital Sky Survey
}

S.K. Leggett国, T.R. Geballe日, Xiaohui Fan日, Donald P. Schneider目, James E. Gunn自, Robert H. Lupton 3, G. R. Knapp3, Michael A. Strauss 3, Alex McDaniel3, David Golimowski5, Todd Henry

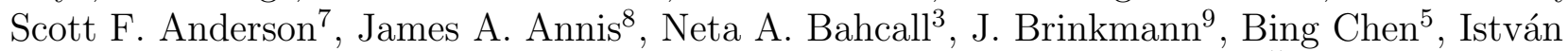
Csabai:10, Masataka Fukugita11 12, G. S. Hennessy 13, Robert B. Hindsley 13, Željko Ivezid3, D.Q. Lamb14, Jeffrey A. Munn15, Jeffrey R. Pier 15 ,David J. Schlege 3 , J.Allyn Smith 16 , Chris Stoughton 8 , A. R. Thakare, and Donald G. York 14

\begin{abstract}
We report the discovery of three cool brown dwarfs which fall in the effective temperature gap between the latest L dwarfs currently known, with no methane absorption bands in the $1-2.5 \mu \mathrm{m}$ range, and the previously known methane $(\mathrm{T})$ dwarfs, whose spectra are dominated by methane and water. The newly discovered objects were detected as very red objects in the Sloan Digital Sky Survey imaging data and have JHK colors between the red L dwarfs and the blue Gl 229B-like T dwarfs. They show both $\mathrm{CO}$ and $\mathrm{CH}_{4}$ absorption in their near-infrared spectra in addition to $\mathrm{H}_{2} \mathrm{O}$, with weaker $\mathrm{CH}_{4}$ absorption features in the $\mathrm{H}$ and $\mathrm{K}$ bands than those in all other methane dwarfs reported to date. Due to the presence of $\mathrm{CH}_{4}$ in these bands, we propose that these objects are early $\mathrm{T}$ dwarfs. The three form part of the brown dwarf spectral sequence and fill in the large gap in the overall spectral sequence from the hottest main sequence stars to the coolest methane dwarfs currently known.
\end{abstract}

Subject headings: brown dwarfs; surveys

\footnotetext{
${ }^{1}$ United Kingdom Infrared Telescope, Joint Astronomy Centre, 660 North A'ohoku Place, Hilo, Hawaii 96720

${ }^{2}$ Gemini Observatory, 670 North A'ohoku Place, Hilo, HI 96720

${ }^{3}$ Princeton University Observatory, Princeton, NJ 08544

${ }^{4}$ Department of Astronomy and Astrophysics, The Pennsylvania State University, University Park, PA 16802

${ }^{5}$ Department of Physics and Astronomy, The Johns Hopkins University, 3701 San Martin Drive, Baltimore, MD 21218, USA

${ }^{6}$ Department of Astronomy, McDonald Observatory, University of Texas, Austin, TX 78712.

${ }^{7}$ University of Washington, Department of Astronomy, Box 351580, Seattle, WA 98195

${ }^{8}$ Fermi National Accelerator Laboratory, P.O. Box 500, Batavia, IL 60510

${ }^{9}$ Apache Point Observatory, P.O. Box 59, Sunspot, NM 88349-0059

${ }^{10}$ Department of Physics of Complex Systems, Eötvös University, Pázmány Péter sétány 1/A, Budapest, H-1117, Hungary

${ }^{11}$ Institute for Cosmic Ray Research, University of Tokyo, Midori, Tanashi, Tokyo 188-8502, Japan

${ }^{12}$ Institute for Advanced Study, Olden Lane, Princeton, NJ 08540

${ }^{13}$ U.S. Naval Observatory, 3450 Massachusetts Ave., NW, Washington, DC 20392-5420

${ }^{14}$ University of Chicago, Astronomy \& Astrophysics Center, 5640 S. Ellis Ave., Chicago, IL 60637

${ }^{15}$ U.S. Naval Observatory, Flagstaff Station, P.O. Box 1149, Flagstaff, AZ 86002-1149

${ }^{16}$ University of Michigan, Department of Physics, 500 East University, Ann Arbor, MI 48109
} 


\section{Introduction}

Brown dwarfs, gravitationally condensed objects whose masses are too low for equilibrium hydrogen burning, occupy the mass range between the lowest mass stars $\left(\sim 0.07 \mathrm{M}_{\odot}\right.$; Burrows et al. 1997) and the giant extrasolar planets $\left(\sim 0.01 \mathrm{M}_{\odot} ;\right.$ Marcy \& Butler 1998). The lowest mass stars and brown dwarfs only slightly cooler than M dwarfs are classified as spectral type "L" (Kirkpatrick et al. 1999, Martín et al. 1997) and have $\mathrm{T}_{\text {eff }}$ in the range $\sim 1500-2000 \mathrm{~K}$. Determining if L-type objects are brown dwarfs is difficult, because the luminosities and effective temperatures of brown dwarfs are a function of both age and mass (e.g. Burrows et al. 1997). The first unambiguous brown dwarf, Gl 229B, was discovered as a companion to a nearby M dwarf by Nakajima et al. (1995). This object is cooler than any $\mathrm{L}$ dwarf and has $\mathrm{CH}_{4}$ in its atmosphere implying $T_{\text {eff }}<1300 \mathrm{~K}$ (e.g. Fegley \& Lodders 1996) and a sub-stellar nature. The recent discoveries of methane dwarfs (tentatively given a "T" spectral clasification) in the field by Strauss et al. (1999), Burgasser et al. (1999, 2000), Cuby et al. (1999) and Tsvetanov et al. (2000) demonstrate that objects like Gl 229B can form singly.

A striking characteristic of known $\mathrm{T}$ dwarfs is the similarity of their spectra, which resemble those of the gaseous solar system planets and are very different from even the coolest L dwarfs. The L dwarf spectra are characterized by the disappearance of the $\mathrm{TiO}$ and VO bands (which are strong in M dwarf spectra), the presence of atomic alkali lines and $\mathrm{CO}$ bands, and increasing depth of the $\mathrm{H}_{2} \mathrm{O}$ bands as $\mathrm{T}_{\text {eff }}$ decreases. The $\mathrm{T}$ dwarfs are characterized by very deep $\mathrm{H}_{2} \mathrm{O}$ and $\mathrm{CH}_{4}$ bands in the $1-3 \mu \mathrm{m}$ region. Alkali metal features are present between the bands, but the $2.3 \mu \mathrm{m}$ $\mathrm{CO}$ bands are absent, due to reduced $\mathrm{CO}$ abundance and the overwhelming strength of the $\mathrm{CH}_{4}$ absorption.

A large remaining gap in the stellar to gas giant planet spectral sequence lies between the previously observed types L and T. In this paper, we report photometry and $0.8-2.5 \mu \mathrm{m}$ spectroscopy of three very red, faint objects identified in the imaging data of the Sloan Digital Sky Survey (SDSS) and observed at the United Kingdom Infrared Telescope (UKIRT) and the Hobby-Eberly Telescope (HET). These objects have spectra intermediate between those of the previously known $\mathrm{L}$ and $\mathrm{T}$ dwarfs, showing the onset of $\mathrm{CH}_{4}$ absorption at 1.6 and $2.2 \mu \mathrm{m}$, while still retaining observable $\mathrm{CO}$ absorption at $2.3 \mu \mathrm{m}$. We propose that they represent the warm end of the "T" spectral sequence, yet to be defined in detail.

\section{Observations}

\subsection{SDSS Photometry and Object Selection}

The three objects described here were selected from SDSS photometric data. SDSS photometry is obtained with a CCD camera at Apache Point Observatory $(\mathrm{APO})$ which images the sky almost simultaneously in five filters: $u^{\prime}, g^{\prime}, r^{\prime}, i^{\prime}$, and $z^{\prime}$ (the data presented here use a preliminary calibration; while we denote the bands by $u^{\prime}$ etc, the magnitudes are denoted by $u^{*}$ etc.). The details of the data acquisition and the photometric and astrometric calibration are described by Gunn et al. (1998), York et al. (2000) and Lupton et al. (2000). SDSS photometry is in the $A B_{\nu}$ system (Fukugita et al. 1996) and the magnitude scale is modified to deal with low signal-to-noise ratios (Lupton et al. 1999).

The $\mathrm{L}$ and $\mathrm{T}$ dwarfs identified in the SDSS (e.g. Strauss et al. 1999, Fan et al. 2000) are undetected in the $u^{\prime}$ and $g^{\prime}$ bands. The L dwarfs have $i^{*}-z^{*}>$ 1.6 while the T dwarfs have $i^{*}-z^{*}>3$. We prepared a candidate $\mathrm{L}$ and $\mathrm{T}$ dwarf list for follow-up observations by searching the SDSS data for point sources with $i^{*}-z^{*}>1.6$. Because of their extremely red colors, some very red, faint objects are detected only in the $z^{\prime}$ band and a search for them can be contaminated by defects. Therefore, additional constraints were imposed: (1) all objects blended with neighbors, or affected by data defects, were removed; (2) an object was required to be detected at $\geq 3 \sigma$ twice: in both $i^{\prime}$ and $z^{\prime}$, in two observations of that region of sky, or detected in the 2MASS database; (3) a $z^{\prime}$-bandonly single detection was required to have $z^{\prime}<19.0$. The three objects discussed in this paper were selected from a total sky area of $225 \mathrm{deg}^{2}$, giving an approximate and very preliminary surface density similar to the 1 per $75 \mathrm{deg}^{2}$ for SDSS T dwarfs found by Tsvetanov et al. (2000).

Table 1 gives, for the three new objects, the J2000 position (accurate to $00^{\prime \prime} 2$ ) and $\mathrm{AB}$ magnitudes at $i^{\prime}$ and $z^{\prime}$. Table 1 also lists photometry obtained for the L dwarf SDSS 0539 (Fan et al. 2000). Hereafter we identify the objects by the first four digits of their Right Ascension. SDSS 0837 was found in two SDSS runs, 1999 March 21 and 2000 February 8; the $z^{*}$ values agree to $0.1 \mathrm{mag}$ and the positions to 
TABLE 1

Coordinates AND Photometry

\begin{tabular}{|c|c|c|c|c|c|c|c|c|c|}
\hline \multicolumn{2}{|c|}{$(2000)$} & \multicolumn{2}{|c|}{$(\mathrm{AB})$} & \multicolumn{3}{|c|}{ (UKIRT-UFTI) } & \multicolumn{3}{|c|}{ (UKIRT-IRCAM) } \\
\hline 053951.99 & -005902.0 & $19.04 \pm 0.02$ & $16.73 \pm 0.01$ & $13.85 \pm 0.03$ & $13.04 \pm 0.03$ & $12.40 \pm 0.03$ & 13.94 & 0.97 & 0.53 \\
\hline 083717.21 & -000018.0 & $(23.51 \pm 0.42)$ & $19.95 \pm 0.09$ & $16.90 \pm 0.05$ & $16.21 \pm 0.05$ & $15.98 \pm 0.05$ & 17.08 & 0.93 & 0.11 \\
\hline 102109.69 & -030420.1 & $(23.73 \pm 0.58)$ & $19.28 \pm 0.05$ & $15.88 \pm 0.03$ & $15.41 \pm 0.03$ & $15.26 \pm 0.05$ & 16.12 & 0.74 & 0.08 \\
\hline 125453.90 & -012247.4 & $22.22 \pm 0.28$ & $18.00 \pm 0.04$ & $14.66 \pm 0.03$ & $14.13 \pm 0.03$ & $13.84 \pm 0.03$ & 14.90 & 0.84 & 0.15 \\
\hline
\end{tabular}

Note. $-i^{*}$ and $z^{*}$ are asinh magnitudes on the AB system (Lupton et al. 1999). Zero flux corresponds to $i^{*}=23.89$ and $z^{*}=22.47$.

$0{ }^{\prime \prime} 3$. SDSS 1021 is a $z^{\prime}$-only detection in data from 2000 February 12 and is also in the 2MASS database. The brightest object, SDSS 1254, has $z^{*}=18.0$ and was found in data taken on 2000 February 2. Finding charts for the new objects are shown in Figure 1.

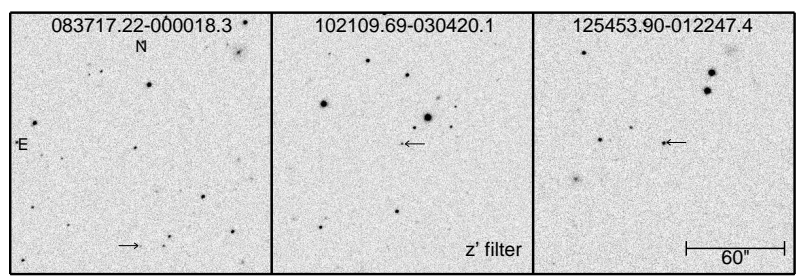

Fig. 1.- Finding charts for the new early $\mathrm{T}$ dwarfs.

\subsection{UKIRT Photometry}

$\mathrm{J}, \mathrm{H}$ and K photometry was obtained using UKIRT's near-infrared camera, UFTI, for SDSS 0837 and SDSS 1254 on 2000 March 2 and for SDSS 0539 and SDSS 1021 on March 14. Both nights were photometric with seeing $\sim 0$ "' 8 . The results are given in Table 1 in the UKIRT (not AB) system - we give JHK on the UFTI system as well as colors on the established IRCAM system for comparison to earlier work. Figure 2 shows a composite color-color plot containing the data from Table 1 and, for comparison, a sample of late M and L dwarfs as well as the SDSS T dwarfs SDSS 1624 and SDSS 1346 (Strauss et al. 1999; Tsvetanov et al. 2000), whose spectra closely resemble that of Gl 229B. Lower limits are shown for $i^{*}-z^{*}$ for the $z^{\prime}$-only detections. The late $\mathrm{M}$ and $\mathrm{L}$ dwarfs show a steady progression towards redder $\mathrm{J}-\mathrm{K}$ and $i^{*}-z^{*}$ colors with later spectral type, however the $\mathrm{T}$ dwarfs have redder $i^{*}-z^{*}$ colors and bluer $\mathrm{J}-\mathrm{K}$ colors due to the strong absorption by $\mathrm{CH}_{4}$ in the $\mathrm{H}$ and $\mathrm{K}$ bands. The $\mathrm{J}-\mathrm{K}$ colors of the new objects lie between those of the previously known L and T dwarfs.

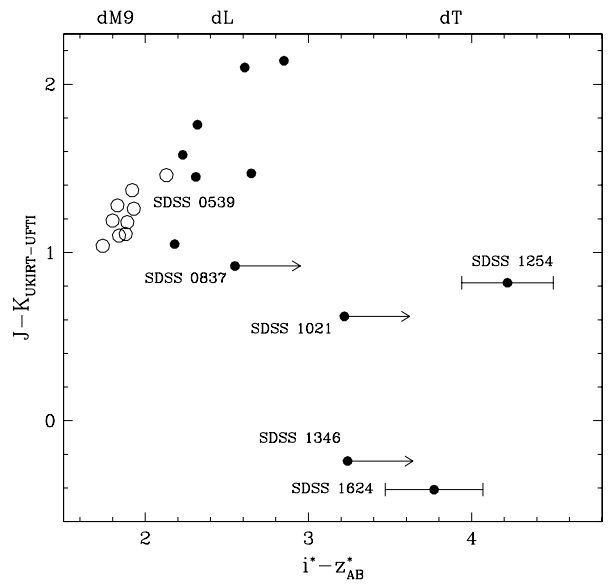

Fig. 2.- SDSS $i^{*}-z^{*}$ against UKIRT-UFTI J-K; open symbols have $\mathrm{J}-\mathrm{K}$ from 2MASS. Errors in $i^{*}-z^{*}$ are $\leq \pm 0.15$ mag except where indicated; measurement error and system differences in the 2MASS J-K are $\sim 0.2$ mag; error in the UKIRT $\mathrm{J}-\mathrm{K}$ is 0.05 mag.

\subsection{HET Spectroscopy}

A red spectrum of SDSS 1254 was obtained on 2000 February 29 with the Low Resolution Spectrograph (LRS; Hill et al. 1998a,b, Schneider et al. 2000) at the prime focus of the of the HET (Ramsey et al. 1998). The spectrum covers the wavelength range $5100-9800 \AA$ at a resolution of $\sim 20 \AA$, but no flux is detected below $\sim 6500 \AA$. 


\subsection{UKIRT Spectroscopy}

Spectra of the objects in Table 1 were obtained in the J, H and $\mathrm{K}$ bands at UKIRT on 2000 February 28 - March 1 and March 13-15 using the facility spectrograph CGS4 (Mountain et al. 1990) at R 400. Integration times were $30-60$ minutes per band. Telluric absorption was removed and the broad-band spectral shapes corrected by comparison with spectra of bright $\mathrm{F}$ stars (with photospheric lines removed) taken immediately before and/or after each observation. The spectra in each band were scaled to match the UKIRT magnitudes using the UFTI filter profiles. UKIRT $\mathrm{z}$-band spectra were also obtained for SDSS 1254 and SDSS 0539. For SDSS 1254 the HET spectrum is used for $\lambda<0.84 \mu \mathrm{m}$, and for SDSS 0539 the red APO spectrum (Fan et al. 2000) is used for $\lambda<0.89 \mu \mathrm{m}$.

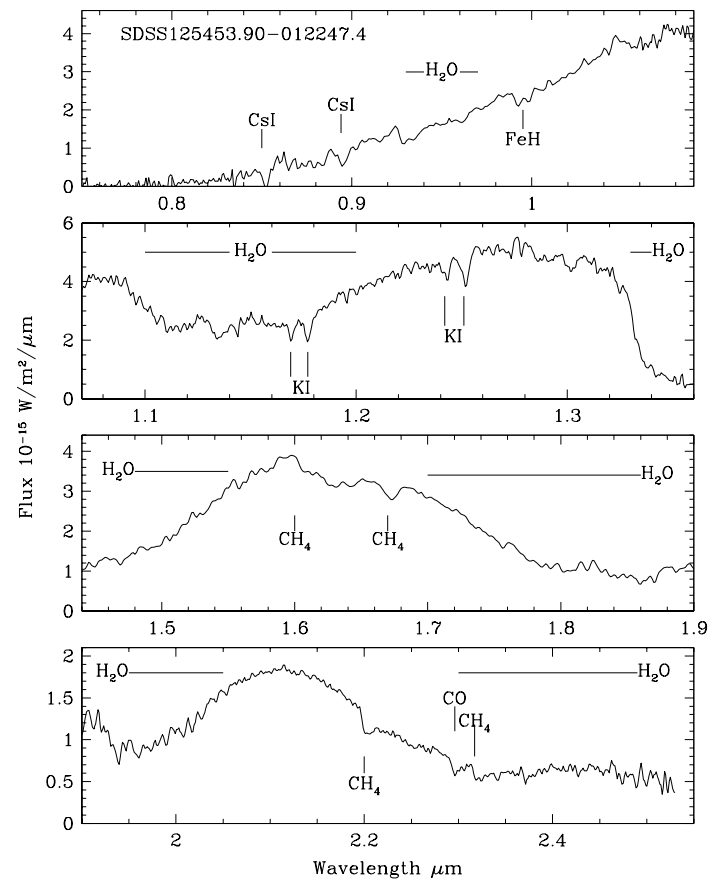

Fig. 3.- UKIRT z, J, H and K spectra of SDSS 1254. The $\mathrm{z}$ spectrum (top) is extended to shorter wavelengths by the HET spectrum. The data are scaled to absolute values using UKIRT photometry. Atomic lines (Cs I, K I), narrow molecular features $\left(\mathrm{FeH}, \mathrm{CH}_{4}\right)$, and bandheads $\left(\mathrm{CH}_{4}, \mathrm{CO}\right)$ are indicated by vertical lines while the broad $\mathrm{H}_{2} \mathrm{O}$ bands are indicated by horizontal lines.

The final flux-calibrated spectra are shown in Figures 3 and 4 . Figure 3 shows the spectrum of SDSS
1254 split into four panels, with the main absorption features marked. Figure 4 compares the spectra of the new objects with those of the L5 dwarf SDSS 0539 (Fan et al. 2000) and the T dwarf SDSS 1624 (Strauss et al. 1999). The spectra show: increasingly red colors at $\lambda<1 \mu \mathrm{m}$, probably due to pressure broadened $\mathrm{Na} I$ and K I absorption (Burrows et al. 2000) combined with decreasing $\mathrm{T}_{\text {eff }}$; increasing absorption in the $\mathrm{H}_{2} \mathrm{O}$ bands at $1.15 \mu \mathrm{m}, 1.4 \mu \mathrm{m}$ and $1.9 \mu \mathrm{m}$; increasing absorption at 1.6-1.7 $\mu \mathrm{m}$ and longward of $2.2 \mu \mathrm{m}$ by $\mathrm{CH}_{4}$ combination and overtone bands; decreasing absorption in the $\mathrm{CO} 2.3 \mu \mathrm{m}$ band. In particular, the absorption maxima of the $\mathrm{CH}_{4} 2 \nu_{2}+\nu_{3}$, $2 \nu_{2}$, and $\nu_{2}+\nu_{3}$ bands at $1.63,1.67$, and $2.20 \mu \mathrm{m}$ become progressively deeper. The CO $2-0$ bandhead at $2.294 \mu \mathrm{m}$ is seen in the new objects, but the $3-1$ bandhead at $2.323 \mu \mathrm{m}$ is absent; it is considerably weaker at these temperatures and also is overwhelmed by the strong $\mathrm{CH}_{4} \nu_{3}+\nu_{4}$ absorption at $2.315 \mu \mathrm{m}$.

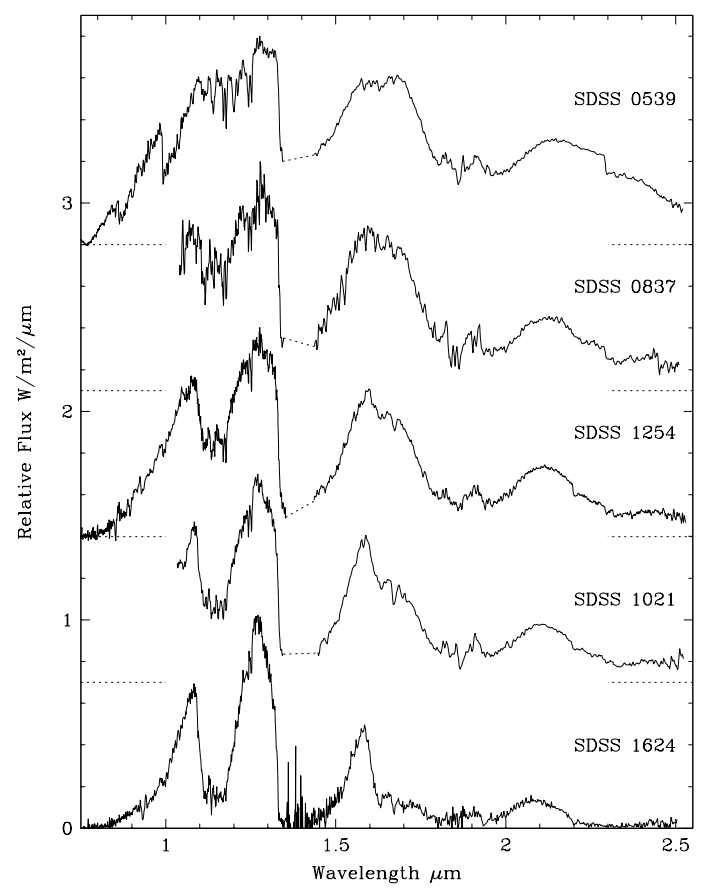

Fig. 4.- A T dwarf spectral sequence. The spectra of the three early T dwarfs (SDSS 0837, SDSS 1254 and SDSS 1021) are ordered by increasing $\mathrm{CH}_{4}$ absorption. Also shown are the L dwarf SDSS 0539 ( L5, Fan et al. 2000, optical data from Fan et al.) and the later T dwarf SDSS 1624 (Strauss et al. 1999). No data were obtained near $1.4 \mu \mathrm{m}$ due to telluric $\mathrm{H}_{2} \mathrm{O}$ absorption. The spectra are scaled to the flux peak at $1.27 \mu \mathrm{m}$ and offset; dotted lines indicate zero levels. 


\section{Discussion and Conclusions}

The spectra in Figure 4 reveal a clear spectral sequence. Thus SDSS 0837, SDSS 1254 and SDSS 1021 are examples of the sought after "L/T transition" objects. However, as suggested by Figure 4, they might more properly be regarded as examples of early T dwarfs, where the " $\mathrm{T}$ " spectral type is defined by the presence of $\mathrm{CH}_{4}$ absorption in the $\mathrm{H}$ and $\mathrm{K}$ bands. The strongest short wavelength infrared band of $\mathrm{CH}_{4}$ is the $\nu_{2}$ band, centered at $3.3 \mu \mathrm{m}$, and objects in which this band is present but the shorter wavelength bands reported here are absent must exist over a narrow temperature range. However, measurements at $3.3 \mu \mathrm{m}$ are considerably more difficult from the ground than are $\mathrm{H}$ and $\mathrm{K}$ band measurements, and objects which show only the $3.3 \mu \mathrm{m} \mathrm{CH}_{4}$ band perhaps should be classified as L rather than $\mathrm{T}$ (Kirkpatrick et al. (1999) suggest that the T class be defined by the presence of $\mathrm{CH}_{4}$ absorption in the $\mathrm{K}$ band). As the three objects presented here are at the $\mathrm{CO} / \mathrm{CH}_{4}$ transition temperature they are likely to have $\mathrm{T}_{\text {eff }} \approx 1300 \mathrm{~K}$ (Fegley \& Lodders 1996) and therefore masses of 20-70 $\mathrm{M}_{\text {Jupiter }}$ for ages in the range 0.3 - 5 Gyr (Burrows et al. 1997).

Figure 2 shows that SDSS easily identifies T dwarfs by their extremely red $i^{*}-z^{*}$ color, including the early $\mathrm{T}$ dwarfs identified in the present paper. On the other hand, the $\mathrm{J}-\mathrm{K}$ colors are similar to those of the common $\mathrm{M}$ dwarfs and these early $\mathrm{T}$ objects are thus very difficult to select on the basis of the nearinfrared colors alone (although the later $\mathrm{T}$ dwarfs are blue and can be selected this way). Many more L and $\mathrm{T}$ dwarfs will be found in the SDSS imaging data.

The SDSS is a joint project of the University of Chicago, Fermilab, the Institute for Advanced Study, the Japan Participation Group, Johns Hopkins University, Max-PlanckInstitute for Astronomy, Princeton University, United States Naval Observatory, and the University of Washington. The site, Apache Point Observatory, is operated by the Astrophysical Research Consortium. Funding has been provided by the Alfred P. Sloan Foundation, the member institutions, NASA, NSF, the U.S. DoE, and Monbusho Japan. The SDSS Web site is at http://www.sdss.org/. UKIRT is operated by the Joint Astronomy Centre on behalf of the UK Particle Physics and Astronomy Research Council. We thank Tim Carroll for his expert and cheerful help with the UKIRT observations, and M.-C. Liang for assistance during the $28 \mathrm{Feb}-$ March 1 run. The Hobby-Eberly Telescope is a joint project of the University of Texas at Austin, Pennsylvania State University, Stanford University, Ludwig-Maximillians-Universität München, and Georg-August-Universität Göttingen. The HET principal benefactors are William P. Hobby and Robert E. Eberly. We thank Phillip MacQueen, Grant Hill, Matthew Sheltrone, and Marsha Wolf for help with the HET data. XF and MAS acknowledge support from the Research Corporation, NSF grant AST96-16901, the Princeton University Research Board, and a Porter O. Jacobus Fellowship. GRK is grateful for support from Princeton University and from NASA via grant NAG6734. DPS thanks the NSF for support via grant AST99-00703.

\section{REFERENCES}

Burgasser, A.J. et al. 1999, ApJ, 522, L65

Burgasser, A.J. et al. 2000, AJ (in press: astro-ph/0004239

Burrows, A., Marley, M.S., \& Sharp, C.M., 2000, ApJ, 531,438

Burrows, A. et al. 1997, ApJ, 491, 856

Cuby, J.G., Saracco, P., Moorwood, A.F.M., D'Odorico, S., Lidman, C., Comerón, F., \& Spyromilio, J. 1999, A\&A, 349, L41

Fan, X. et al. 2000, AJ, 119, 928

Fegley, B. \& Lodders, K., 1996, ApJ, 472, L37

Fukugita, M., Ichikawa, T., Gunn, J.E., Doi, M., Shimasaku, K., \& Schneider, D.P. 1996, AJ, 111, 1748

Gunn, J.E. et al. 1998, AJ, 116, 3040

Hill, G.J., Nicklas, H.E., MacQueen, P.J., Tejada, C., Cobos Duenas, F.J., \& Mitsch, W. 1998a, Proc. SPIE, 3355,375

Hill, G.J., Nicklas, H.E., MacQueen, P.J., Mitsch, W., Wellem, W., Altmann, W., Wesley, G.L., \& Ray, F.B. 1998b, in Proc. SPIE, 3355, 433

Kirkpatrick, J.D. et al. 1999, ApJ, 519, 802

Lupton, R.H., Gunn, J.E., \& Szalay, A. 1999, AJ, 118, 1406

Lupton, R.H. et al. 2000, in preparation

Marcy, G.W., \& Butler, R.P. 1998, in "Brown Dwarfs and Extrasolar Planets", ed. R. Rebolo, E.L. Martín \& M.R. Zapatero-Osorio, A.S.P. Conf. Ser. 134, 128

Martín, E.L., Basri, G., Delfosse, X., \& Forveille, T. 1997, A\&A, 327, L29

Mountain, C.M., Robertson, D., Lee, T.J., \& Wade, R. 1990, Proc. SPIE, 1235, 25

Nakajima, T., Oppenheimer, B.R., Kulkarni, S.R., Golimowski, D.A., Matthews, K., \& Durrance, S.T., 1995, Nature, 378,463

Ramsey, L.W. et al. 1998, Proc. SPIE, 3352, 34

Schneider, D.P. et al. 2000, PASP, 112, 6

Strauss, M.A. et al. 1999, ApJ, 522, L61

Tsvetanov, Z.I. et al. 2000, ApJ, 531, L61

York, D.G. et al. 2000, submitted to AJ

This 2-column preprint was prepared with the AAS LATEX macros $\mathrm{v} 4.0$. 